U. S. DEPARTMEITT OF COMNERCE

NATIONAL BUREAU OF STANDARDS

WASHINGTON, D. C.

LIST OF COMMERIAI, STANDARDS

Revised to January 2,1941

\title{
GENERAL INEORNATION
}

Where the price is stated, the publication can be purchased from the Superintendent of Documents, Government Printing Office, Washington, D. C. The prices quoted are for delivery to addresses in the United. States and its territories and possessions and in certain foreign countries which exteno the franking privilege. In the case of all other countries, one-thind the cost of the publication should be added to cover postage. Remittances should be made either by coupons (obtainable from the suporintendent of Documents in sets of 20 for $\$$ l. 00 and good until used), or by check or money order payable to the "Superintendent of Documents, Government Printing Ofilce, Washington, D. C." and sent to him with orüer.

A discount of 25 percent will be allowed on orders for 100 or more conies.

Those marked "OP" are out of print, but, in ceneral, are mineographed or may be consulted at technical libraries or many public libraries.

Mineographed copies of Comercial Stanciards are avallable without charge, except those marked IN (Not Nimeographea), from the Division of Trade Standards, National Bureau of Standaras, Washington, D. C.

\section{Title}

Adverse-weather lamos ior veinicles (after mariset) Apple wraps Bathroom accessories, colors for - - . Blankets, wool and part-wool (second edition) Blanks, care (thirc edition) - an) $--\cdots-$ Blown, draw end aropoed Ienses for sun glasses (second. edition) Board; binders, for bookbinding and other purposes _ _ _ _ _ Board; chip, laminated chip board and miscellaneous boards for bookbinding purposes _ . . . . . . . $49-34$
CS IIO

Price

$81-41$

$44-32$

$63-36$

$\begin{array}{ll}5 & 6 \\ 5 & 6 \\ 5 & 6\end{array}$

$30-37$

$8-41$

$61-37$

$5 \not$

Mimeographed

$5 \notin$

79-40 Mimeographed

$50-34$

$5 \not$

$5 \not$ 
Board; fiber insulating (secona ecition)- - - 42-35 Bone plates, steel and screws - - - M - - 37-31 Book cloths, buckrans and impregnated

fabrics for bookbinaing purposes except

library binaings (second eaition) - - - - 57-40 Burners, domestic, for Pennsylvania

anthracite (underfeed type)(second edition)-48-40 Burners, autoratic mechanical draft, oil - - 75-39 Cast stone, colors and finishes for - - - - 53-35 Chip boara, laminated chip boarả anà

miscellaneous boaràs lor bookbinding

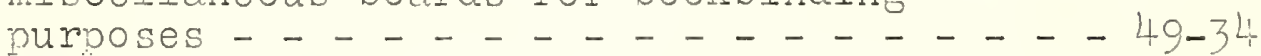
Clearance, marker and icuentification

lamps for vehicles (after narket) - - - - 83-41 cloth, cotton, for mbiber ano.

pyroxylin coating _ _ . . . . . . - 32-31 cloth, wire, fourdrinier

(secono edition) - - - - - - NM- - - 36-33 Clotis, book; buckrams and impregnated.

Iabrics Ior bookbinding purposes except

Iibrary bindings (second edition) - - - - 57-40 Coal tar disinfectant (emulsifying type) - - 70-38 Colors and finishes ior cast stone - - - - 53-35 Colors for bathroom accessories - . - Colors for kitchen accessories - - - - - $62-38$ Colors for sanitary ware _ _ _ _ - . - - 30-3I Commercial standards and their value to

business (thira edition) _ _ _ _ NH. _ - 0.40 Covers, cotton Iabric, tents anci

tarpaulins _ . . . . . . . . . - - 28-32 Cresylic disingectants _ _ - . - - - $71-38$ Direction signal systems; electicic, other

than semaphore type for commercial and.

other vehicles subject to special motor

vehicle laws (after narket) _ _ . - . - $80-41$ Disinfectant; coal tar (emulsifing type) - - 70-38 Disinfectants; cresilic _ _ . _ . . - . - 7.l-3S Disinfectant, deodornt and germicide;

Iiquid hypochlorite _ - . - . - . - $683-38$ Disiniectant; pine oil - - . - . - - 69-38 Doors; old growth Douglas fir

standard stock (with amendment) - _ - - 73-38 Douglas fir plywooa (aomestic gracies)

(fourth edition) - _ - _ - . - . - $45-40$ Dress fabrics, roven - testing

ana reporting (seconj eāition) - - - - - 59-39

Dress patterns (second edition) - - - . - 13-39

Drill fittings, diamono cone

(second edition) - _ _ - _ - m - - 17-32

Dry cleaning solvent (stoädrra

solvent) (thirā eảition) _. . . . . - 3-40

Enameled ware; sanitary cast iron - - - - $77-40$
$5 \%$
$10 \%$

$5 \not$

IIneosraphed

$5 \notin$

$5 \not$

$5 \notin$

$5 \not$

5 c

$5 \phi$
$5 \phi$
$5 \phi$
$5 \phi$
$5 \phi$
$20 \phi$

No cinarge

$\mathrm{OP}$

$5 \not$

$5 \phi$
$5 \phi$

$5 \not$

$10 \not$

$5 \not$

5

$5 \varnothing$

$5 \notin$ 
List of Commercial Standards - 3

Title

Fabrics, mohair pile - - - - - - - - 52-35

Fabrics, wool and part-wool _ . . . - - 65-38

Fabrics, woven cress -

testing and reporting (second edition) - -59-39

$5 \notin$

Fabrics, woven elastic, for use in

overalis (overall elastic webbing) - - - 58-36

Felaspar _ _ _ _ _ _ _ _ - M _ - 23-30

Fir standard stock doors;

old. Growth Douglas (with amenament) - - - 73-38

Flares; liquid-burning _ _ _ . . . . - 88841

Flares, see red electric warning lanterns- - 87-41 Flooring, oak (second edition) - - - - - 56-41

Fog lamps, see adverse-weether lamps

for vehicles (after mariset) - _ - . - $81-41$

Fuel oils (fifth eaition) - . - . - - 12-40

Gage blanks (thirā eàition) - . . . - 8-41

Gloves, latex, surgeons - - . - - - - 41-32

Gloves, rubber, surgeons' - _ - - - $-40-32$

Gold filled and rolied gold plate articles,

other than watchcases, markine of

(with supplement) _ _ _ _ _ . . . - 47-34

Gold, karat; marking articles made of - - $-67-38$ Golf shafts, hickory - _ - _ - Ni _ - I8̈-29 Ground-and-polishea lenses for

sun glasses (second edition) - - . - - 78-40 Haraware, huilders' (nontemplate)

(seconá edition) _ _ _ . . . . - 22-40

Hardware, builders! template

(second'edition) - - - - . - . - - $9-33$

Hardwood dimension lumber - - - - - - 60-36

Hardwood interior trim and molding - - - - 76-39

Hardwood stair treads and risers - - - - - 89-40

Hardwood wall paneling; solid - - - - - 74-39

Hosiery lengths and sizes (thira edition) - 46-40

Househola insecticice (Iiquid spray type) - 72-38

Hypochlorite (Iiquid) disinfectent,

deodorant and Germicide _ _ . . . - - - 58-38

Insecticide; household (Ilduid spray type) - 72-38

Joints, ground-glass, interchangeable,

stopcocks and stoppers (fourth edition) - - 2I-39

Kitchen accessories, colors for - _ - - - 62-38

Lamps; adverse-treather, for vehicles

(after market) - _ _ _ . _ _ . - - 3I-4I

Lamps; clearance, marker and identilication, for vehicles (after market) - _ _ . - - $33-41$

Lamps; electric Iicense-plate, for vehicles (after market) - _ _ . - . - 85-41

Lamps; electric stop, for vehicles

(after market) _ _ _ _ _ _ . . $86-41$

$5 \not$

$5 \not$

$10 \not c$

$5 \not$

Mimeographed

$5 \not$

Mimeographed

${ }^{5} \not \subset$

$5 \phi$
$5 \phi$
$10 \phi$

Mimeographed

$10 \not$

$5 \phi$
$10 \phi$
50
$5 \phi$
$5 \phi$
$5 \phi$
$5 \phi$

$5 \not$

$5 \not$

$5 \not$

$5 \varnothing$

$5 \not$

Lamps; electric tail, for vehicles

(after market) _ _ _ _ _ _ . . . - $84-41$

Lanterns; red electric worning _ . . . . 87-41

$5 \varnothing$

$5 \not$ 
List of Commercial Standards - 4

iste

Price

Leather, bag, case and strap - . . . - 34-3I

$5 \not$

Lenses for sun glasses; blown,

drawn and dropped (second edition)

Lenses for sun glasses; Ground-and-

polished (second edition) _ . . . . . $78-40$

License-plate lamps; electric, for

vehicles (after market) - _ . . . . - $85-41$

Lining, closet, red cedar (eromatic) - - - 25-30

Lumber, hardwoóa dimension _ . . . . - - 60-36

Marker and identification lamps; clearance,

for vehicles (after marlet) - . . . . - $83-4.1$

Marking articles mace of karat gold - - - 67-38

Marking of articles made wholly or

in pert of platinum - . . . . . . - 66-38

Varking of articles made of silver

in combination with gold - . - . - - 51-35

Marking of gola filled and rolled gola

plate articles other than watchcases

(with supplement) _ . . . . . . . - 47-34

Mattresses for hospitals … - . - . - 54-35

Mattresses for institutions - - . - - $55-35$

Mirrors (second edition) - . - . - - 27-35

Mohair ptle fainics - . . . . - - $52-35$

Mopsticks _ . . . . . . . . . 2-30

Nipples, pipe; brass, copper steel, and.

wrought iron (Revision and consolidation

of CS5-29, CS6-31, and (SIO-29) - . - .

oak flooring (eoond edition) . . . . . - 56-4I

Oil burners; automatic mechanical craft - $75-39$

Oils, fuel (fifth edition) - . . . . - 12-40

Oils, sulphonated, grading of - . . - 43-32

Pajamas, men's _ - . - . . - - - 15-29

Paneling, solid hardwood wall … - - $74-39$

Paper, waII _ . . . . . . . - - 16-29

Patterns, dress (second edition) - - - - 13-39

Patterns, founäry, wood (seconc edition) - - 19-32

Pine oil disinfectant _ . . . . . . - 69-38

Pipe nipples; brass, copper, steel, and

wrought iron (Revision cnd consolidation

of CS5-29, CS6-31, and CS10-29) - - - - 5-40

Platinum; marking of articles made

wholly or in part of $\ldots \ldots \ldots-\ldots 6-38$

Plumbing fixtures, staple porcelain

(aIl-clay ) _ . . _ _ . . . . . 4-29

Plumbine fixtures, staple vitreous

china (second edition) - . . . . - 20-36

Plywood, Douglas fir (donestic gracies)

fourth edition) - . . . . . . - $45-40$

Plywood (hardwood and eastern red cedar) - - 35-31

Porcelain (a.l-clay) plumbing

fixtures, staple - . . . . . . $4-29$

Sanitary cast iron enameled. ware - . - . $77-40$

Screw threads, special - - - - M - - 25-30

Screw threads, standard - - - N - - $24-30$

Mimeographed.

Mimeographed

$\begin{array}{rl}5 & \varnothing \\ O P & 0\end{array}$

$10 \not$

$5 \not$

$5 \not c$

$5 \not$

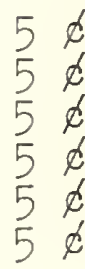

$5 \not$

Mimeographed

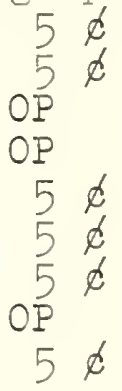

$5 \not$

$5 \not$

$O P$

$10 \not$

OP

OP 
List of Commercial Standards - 5

Title

Seats for water closet bowls, staple - - Shafts, golf, hickory - - - - - NM - Sheeting, rubber (hospital) - - - - - Shingles, wood (fourth edition) - . - . Shirts, junior and polo sinirts; boys' button-on waists (second eaition) - - - Signal systems; direction; electric, other than semaphore type for commercial and other vehicles subject to special motor vehicle laws (after mariset) $-\overline{-}$
silver in combination with gold, marking of articles made of - - . - - 51-35 Solvent, stoddard (ary cleaning)

third edition) _ . . . . . .

Spotlamps; inner-controlled, for vehicles

(after market) - - - . - - - - - - $82-41$

Stair treads and risers, haromood - . - . $89-40$ Staple vitreous china pluraing fixtures

(second edition) - _ _ . _ _ . . . - 20-36 Stokers, domestic (see burners for

Pennsylvania anthracite) (seconá eaition) - - 4s-40 Stone, cast, colors and finishes for - - - 53-35 Stop lamos; electric, for vehicles

(after market) - - - _ - - - . - - $36-41$

Stopcocks, interchangeable ground-glass,

stoppers ano joints (fourth eajtion) - - 21-39

Sulphonated oils, grading of _ _ . . . - 43-32

Tail lamps; electric, for vehicles

(after market) _ - _ . . . . . . . -

Tents, cotton fabric, tarpaulins and covers -

Themometers, clinical (second edition) - -

Treads and risers, hardwood stair - - - -

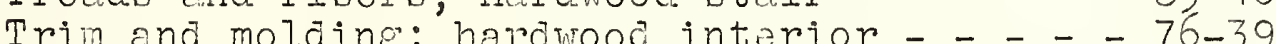

Underwear, knit (exclusive of rayon) - - - 33-32

Unions, malleable iron or steel,

screwed, standard weight _ _ - . - . - 7-29

Veneers, walnut _ _ _ _ . . . . . - - $54-37$

Venetian blinds, wood-slat _ . . - . - 61-37

Vitreous china plumbing fixtures, staple

(second eaition) _ _ _ _ _ _ _ . - 20-36

Waists, boys' button-on; shirts,

junior and polo shirts (second edition) - 14-39

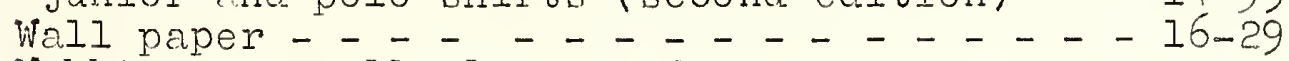

Webbing, overall elastic, for use in wire cloth, fourdrinier (seconde é̄ition) $\bar{N}-58-36$

Wool and part-wool blankets (second edition.)-

Wool and part-Wool fabrics _ _ . . . . . -

Woven aress fabrics - testing and

reporting (second edition) - - - . - - 59-39

Woven elastic fabrics for use in

overalls (overall elastic webbing) - - - $-58-36$

Wraps, apple - - _ - _ - _ - NM - $44-32$

Yerns, mercerized cotton,

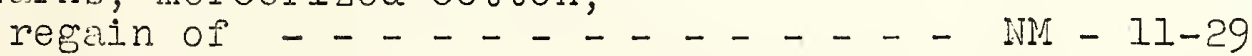

Price

$5 \not$
$10 \not$
$5 \not$
$5 \not$

$5 \not$

$5 \not$

$5 \not$

$5 \not c$

$5 \not$

$10 \not$

Mimeographed

$5 \notin$

$5 \not$

OP

os

$O P$

$5 \notin$
$5 \notin$
OP

$5 \not$
$5 \not$
$5 \not$

$10 \not$

$5 \not$

$5 \%$
$5 \%$
$5 \%$

$5 \notin$

$5 \notin$

$5 \not$ 


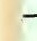

